\title{
Geometrical Interpretation of Isomers
}

\section{Lemi Türker}

Department of Chemistry, Middle East Technical University, Üniversiteler, Eskişehir Yolu No: 1, 06800 Çankaya/Ankara, Turkey; e-mail: lturker@gmail.com; lturker@metu.edu.tr

\begin{abstract}
The present article considers isomerism, which is one of the most important topics of chemistry. A model is proposed in $2 \mathrm{D}$ and $3 \mathrm{D}$-Euclidean geometry starting from the very fundamental concepts and has established certain geometrical relationships between the mass of a molecule and its bonds and atoms. Some crucial angles are defined. Certain mathematical analysis have been presented as well.
\end{abstract}

\section{Introduction}

By definition, isomer is one of two or more molecules having the same number and kind of atoms and hence the same molecular weight but differing in respect to the arrangement of configuration of the atoms [1]. The phenomenon of isomerism is fundamental importance in chemistry, especially in organic chemistry. For over a century, it has dominated organic chemistry and also plays a role in many classes of inorganic compounds especially in organometallic complexes. Due to the directional and localized character of simple covalent bonds, a group of atoms may be linked in several nonisomorphic ways to yield various isomers. Parallel to the development of chemical structural theory, attention of scientists was concentrated to isomers and enumeration of isomers. English scientists Arthur Cayley in 1875 employed the graph theoretical methods to solve the problem of counting isomers of alkanes $\left(\mathrm{C}_{n} \mathrm{H}_{2 n+2}\right)$ [2]. Later on various researchers contributed to enumeration of isomers as well [3].

Traditionally isomerism has been classified as either constitutional or steric with the

Received: July 5, 2021; Accepted: August 3, 2021

Keywords and phrases: isomer, isomerism, structural isomerism, enantiomers, diastereomers.

Copyright (C) 2021 Lemi Türker. This is an open access article distributed under the Creative Commons Attribution License, which permits unrestricted use, distribution, and reproduction in any medium, provided the original work is properly cited. 
later class being subdivided into enantiomers and diastereomers [4]. Isomerism, especially stereoisomerism plays a vital role in pharmacology in terms of structure and activity of molecules. An alternative subdivision was proposed by Mislow who defined isometric and anisometric structures based on the isometry operations [5]. In recent years some theoretical work has appeared also about different aspects of the isomerism [6-9].

However, in the literature, most of the articles about isomerism deal with consequences of isomerism rather than fundamental aspects of the theory of isomerism or isomers.

In the present study, a geometrical model is proposed to put some light on understanding of isomerism.

\section{Theory}

Consider a triangle described by edges $\mathrm{M}, \mathrm{n}$ and e (OM, OA and AM, respectively) (Figure 1). Note that triangles OAM and OA'M are congruent having the same edges ( $\mathrm{OA}=\mathrm{MA}^{\prime}, \mathrm{AM}=\mathrm{OA}^{\prime}$ and $\mathrm{OM}$ is common) and angles [10]. Additionally they possess the same area because they have a common base $\mathrm{OM}$ and the same height $\left(\mathrm{AH}=\mathrm{A}^{\prime} \mathrm{H}^{\prime}\right)$. On the other hand, ODM triangle also shares the same base OM but of course has a different area. It represents a different system.

Now assume that certain isomers are described by these triangles in a certain way.

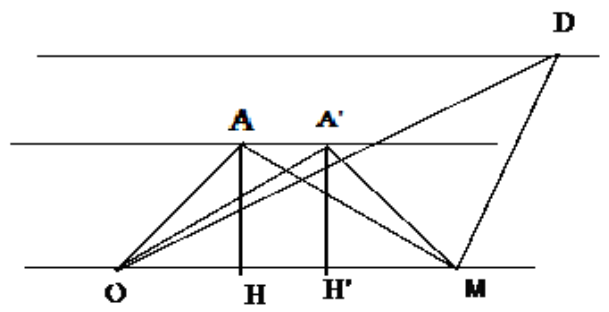

Figure 1. Various triangle sharing the same base OM.

In three dimensional Euclidean space when those triangles OAM or OA'M (each one is half of a deltoid, or parallelogram) revolve about OM they engender certain right circular bicones having the common axis OM and the common base, disc of radius O'A (Figure 2). If $\mathrm{e}=\mathrm{n}$, then the $2 \pi$ revolution process about $\mathrm{OM}$ produces congruent right 
circular bicones in 3-dimensional space. The base of cone is the disc including point A which is the apex of the triangle OAM in Figure 1.

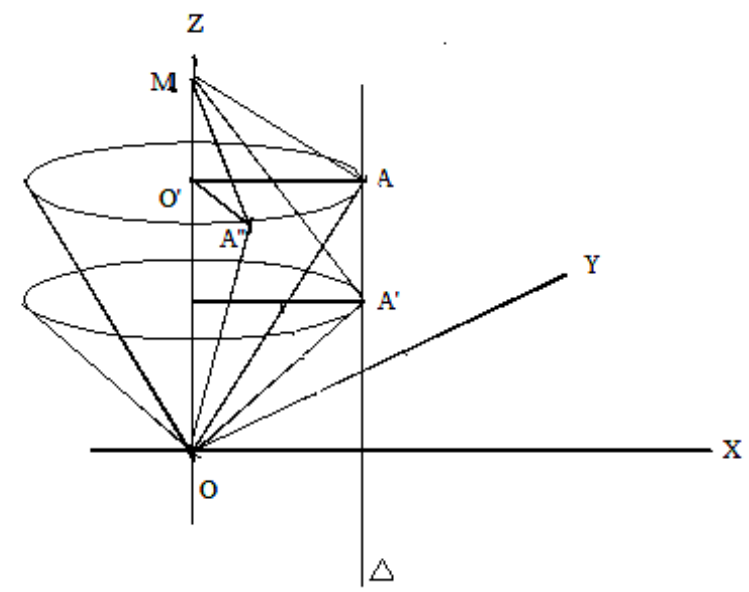

Figure 2. Various systems described by cones having a common axis OM.

Adaptation of the model to molecular systems is possible. Then, Figure 2 shows the situation of various isomers described by bicones having a common axis OM. In Figure 2 the cone OO'A (or OO'A") stands for enantiomers or diastereomers which possess the same $\mathrm{n}$, e and $\mathrm{M}$ values.

Now, construct a triangle such that base (OM) length of $M$ and the side edges are $n$ and e (see Figure 3). Draw a perpendicular to OM from point A. Construct OAK isosceles. Note that $\mathrm{OM}=\mathrm{x}+\mathrm{y}$, and $\mathrm{KM}=\mathrm{y}-\mathrm{x}$. In $\mathrm{OAH}$ and HAM triangles application of Pythagoras theorem yields,

$$
\begin{aligned}
& h^{2}=n^{2}-x^{2} \\
& h^{2}=e^{2}-y^{2}
\end{aligned}
$$

By equating eqs. (1) and (2) and rearranging one obtains eq. (3).

$$
n^{2}-e^{2}=x^{2}-y^{2}
$$

Factorizing the right hand side,

$$
\begin{aligned}
& n^{2}-e^{2}=(x+y)(x-y) \\
& n^{2}-e^{2}=-(x+y)(y-x)
\end{aligned}
$$


Since,

$$
\begin{gathered}
x+y=O M \\
y-x+2 x=O M
\end{gathered}
$$

and $\mathrm{OM}=\mathrm{M}$, eq. (5) becomes eq. (8).

$$
n^{2}-e^{2}=-M(M-2 x)
$$

Solving for $\mathrm{x}$,

$$
\mathrm{x}=\left(\mathrm{n}^{2}-\mathrm{e}^{2}+\mathrm{M}^{2}\right) / 2 \mathrm{M}
$$

Substituting eq. (9) into eq. (1) and solving for $\mathrm{h}^{2}$ one gets,

$$
h^{2}=\left[4 n^{2} M^{2}-\left(M^{2}+n^{2}-e^{2}\right)^{2}\right] / 4 M^{2}
$$

For $\mathrm{h}^{2}$ to be real it requires

$$
4 n^{2} M^{2}-\left(M^{2}+n^{2}-e^{2}\right)^{2} \geq 0
$$

By factorizing,

$$
\left[2 n M+\left(M^{2}+n^{2}-e^{2}\right)\right]\left[2 n M-\left(M^{2}+n^{2}-e^{2}\right)\right] \geq 0
$$

Rearranging the terms in the parentheses ineq. (12) becomes,

$$
\left(M^{2}+2 n M+n^{2}-e^{2}\right)\left(-M^{2}+2 n m-n^{2}+e^{2}\right) \geq 0
$$

Ineq. (13) requires each parenthesis to be positive or both mutually negative,

$$
\begin{aligned}
& M^{2}+2 n M+n^{2}-e^{2} \geq 0 \\
& -M^{2}+2 n m-n^{2}+e^{2} \geq 0
\end{aligned}
$$

Ineq. (14) yields positive values if $-(e+n) \leq M \leq n-e$, whereas ineq. (15) requires that e- $n \leq M \leq e+n$. Since $M$ is positive all the time and $n$ less than or equal to e for classical compounds, the first set of bounds are not meaningful. On the other hand, if both of the parentheses in ineq. (13) are negative, then one of them yields an imaginary value for $\mathrm{M}$ which is absurd. 


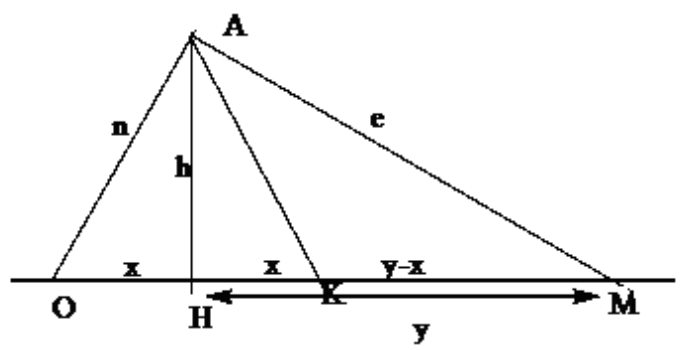

Figure 3. The triangles considered in the derivations.

Consider OAM (Figure 2 or 4) triangle. The cosine theorem yields,

$$
\mathrm{M}^{2}=\mathrm{n}^{2}+\mathrm{e}^{2}-2 \mathrm{en} \cos \lambda
$$

where $\lambda$ is OAM angle (geometrical parallax). Then solving for $\cos \lambda$,

$$
\cos \lambda=\left(\mathrm{n}^{2}+\mathrm{e}^{2}-\mathrm{M}^{2}\right) / 2 \mathrm{ne}
$$

Since, $-1 \leq \cos \lambda \leq 1$ is required, then eq. (17) yields $\mathrm{M}^{2} \geq(\mathrm{n}-\mathrm{e})^{2}$ for $\cos \lambda \leq 1$ and $\mathrm{M}^{2} \leq(\mathrm{e}+\mathrm{n})^{2}$ for $\cos \lambda \geq-1$. These are the boundaries for $\mathrm{M}^{2}$ obtained previously.

On the other hand, note that in the Hückel framework, the total $\pi$-electron energy $\left(\mathrm{E}_{\pi}\right)$ is given by

$$
\mathrm{E}_{\pi}=2 \sqrt{n e} \cos \lambda
$$

for even alternant hydrocarbons having $2 \mathrm{n}$ vertices (atoms) and e edges (bonds) in their $\pi$-skeletons [11]. Let the angle between $\mathrm{n}$ and e be $\lambda$. Solving for $\cos \lambda$ and inserting it in to eq. (18), then rearranging one gets,

$$
\mathrm{E}_{\pi}=\left(\mathrm{n}^{2}+\mathrm{e}^{2}-\mathrm{M}^{2}\right) / \sqrt{n e}
$$

whose molecular graph is represented by $\mathrm{G}(2 \mathrm{n}, \mathrm{e})$.

\section{Results and Discussion}

Note that Figure 2 shows only one of the cones of a bicone. The base of those cones have the same radius although they are lower part of different bicones. The azimuthal MOA and MOA" angles are equal $(\Theta)$. MOA and MOA' angles are different and they stand for different isomers but their base radii $\left(\mathrm{O}^{\prime} \mathrm{A}=\mathrm{r}\right)$ is the same. In a similar fashion another line $\left(\Delta^{\prime}\right)$ parallel to $\mathrm{Z}$ axis and line $\Delta$ could be drawn and some triangles having their apexes on this line and possessing $\mathrm{OM}$ as common edge might exist. They are of 
course isomer of the previously considered cases as the molecular weight considered only, but the triangles whose apexes are on the different lines possess different areas different $\mathrm{n}$ and e values. This type of isomerism is different (see below).

The bicones having e and $\mathrm{n}$ common can be classified as i) isomers of $(\mathrm{r}, \Theta)$ type cones for which radii and azimuthal angle are constant ( $\mathrm{r}$ and $\Theta$ are constant but $\Phi$ is variable) or ii) just radii equivalent ( $r$ ) cones namely $r$ is constant but $\Theta$ and $\Phi$ are variable. In the first group, the triangles OMA and OMA" shown in Figure 2 have the same area. Then as a conjecture, in the first group of bicones (as mentioned above) stereoisomers (enantiomers, diastereomers and meso compounds) topomers or different crystalline forms of the same compound should exist. Note that different crystalline forms of a molecule should be on the same cone but having different angle $\Phi$ (AO'A") (Figure 2). In the second group isomers whose bicones are related to OMA and OMA' triangles which also have the same area but possess different $\Theta$ values (as shown in the figure or even have different $\Phi$ angles). In the third group isomers only $\mathrm{M}$ is common but $\mathrm{r}, \Theta$ and $\Phi$ are different ( $(\mathrm{f}, \Theta, \Phi)$ ). Constitutional isomers may belong to any of these groups depending on their peculiarities.

Figure 4 shows extracted triangles of bicones describing molecules in 2-dimensional

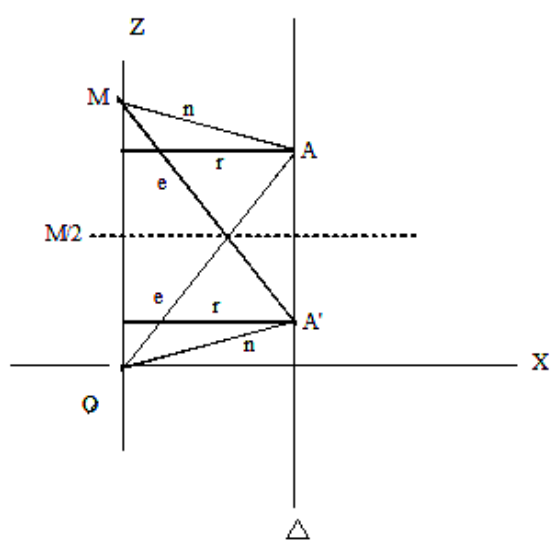

Figure 4. Extracted triangles of a bicone describing enantiomers.

space. As point $\mathrm{A}$ moves towards $\mathrm{A}^{\prime}$ on the director $\Delta$, various triangles having a common edge $\mathrm{OM}$, but different $\mathrm{n}$ and e are engendered (they all have the same area $\mathrm{S}=\mathrm{M}^{2} / 2$ ). They consequently have the same radius ( $\mathrm{r}$ ) values. In the case in which MOA and $\mathrm{OMA}^{\prime}$ angles are equal $(\mathrm{MOA}=\Theta)$ stand also for stereoisomers. They have the same $\mathrm{n}$ and e values. Other triangles stand for other molecules which are not the isomers of the 
previous case because they possess different e and $n$ values, although they have the common edge OM thus the same molecular weight. That is possible only if some centric perturbations occurred in the structure, namely kind of atoms have been changed to keep the same mass while $\mathrm{n}$ and $\mathrm{e}$ values modified. However, note that it may not be chemically possible at all. Various structural factors dictate the properties of molecules. Of these $M, n$, e are responsible for the gross topology of them. The azimuthal angle $\Theta$ in MOA triangle dictates the fine topology of the isomers whereas $\Phi$ (AO'A" angle in Figure 2) should be responsible for higher fine (ultra fine) topology such as stereoisomers or different crystalline forms of a molecule.

Note that when $n=e$, the Euler's formula $e=n+f-2$ (where e, $n$ and $f$ are the number of edges, vertices and faces, respectively [14]) in 2-dimentional case yields,

$$
\mathrm{e}=\mathrm{n}+\mathrm{R}-1
$$

which gives the number of rings $(\mathrm{R})$ as $\mathrm{R}=1$. Thus, a monocyclic structure is obtained for which point $\mathrm{A}$ (Figure 3 ) coincides with a point on the director at $\mathrm{M} / 2$.

In the Hückel molecular orbital (HMO) framework isospectral molecules [12] are constitutional isomers and should belong to the first group isomers. Isospectral molecules possess quite different structures but they have the same molecular orbital energy spectrum, thus have the same total $\pi$-electron energies [13]. They are isomers of $(r, \Theta)$ type, even some of them results in congruent bicones. In higher order molecular orbital treatments, due to structure optimization process involved, they may fall on different types of cones. Nevertheless, there might have some similarities or identities in terms of properties of them.

Figure 5 shows a pair of isospectral molecules in the Hückel framework. Note that they are not isomorphic.
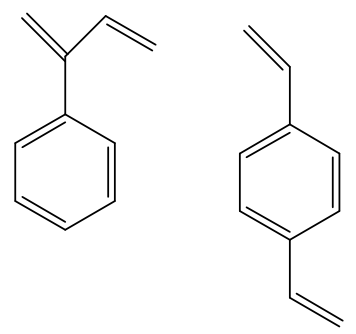

Figure 5. A pair of isospectral molecules of the Hückel framework. 
For chemical structures, it is $\mathrm{e} \geq \mathrm{n}$ because of the Euler's formula, $\mathrm{e}=\mathrm{n}+\mathrm{R}-1$ [14]. In general most of the cases magnitude of $\mathrm{M}$ is not comparable with magnitude of $\mathrm{n}$ and/or e. Then, it is possible either adopting a suitable unit for $\mathrm{M}$ or scaling down its magnitude to have comparable numbers.

\section{Conclusion}

The modeling proposed and analyzed presently has established a geometrical presentation of the isomerism, based on invariants of a molecule namely its mass, numbers of its bonds and atoms. The interrelationship existing between the isomers has been explained in terms of certain presently defined angles. Certain trigonometric relations direct the angles $\Theta$ and $\Phi$. Thus determine the relative positions of the isomers on the base circle of the bicones considered.

\section{References}

[1] G.G. Hawley, The Condensed Chemical Dictionary, 9th ed., New York: van Nostrand and Reinhold, 1977. https://doi.org/10.1080/00945717708069741

[2] I.S. Dimitriev, Molecules Without Chemical Bonds, Moscow: Mir, 1981.

[3] N. Trinajstic, Chemical Graph Theory, 2nd ed., Boca Raton: CRC Press, 1992.

[4] D. Bonchev and D.H. Rouvray, Chemical Graph Theory, New York: Gordon and Beach, 1991.

[5] K. Mislow, Bull. Soc. Chim. Belges 86 (1977), 595-601. https://doi.org/10.1002/bscb.19770860805

[6] S. Zdenek, Contemporary Theory of Chemical Isomerism, Amsterdam: Springer Netherlands, 1986.

[7] A. Ugulava, Z. Toklikishvili, S. Chkhaidze, R. Abramishvili and L. Chotorlishvili, Quantum theory of rotational isomerism and Hill equation, Journal of Mathematical Physics 53(6) (2012), 062101. https://doi.org/10.1063/1.4729247

[8] P.R. Remya and C.H. Suresh, Theoretical evidence for bond stretch isomerism in Grubbs olefin metathesis, Journal of Computational Chemistry 38(19) (2017), 1704-1711. https://doi.org/10.1002/jcc.24814

[9] P.C. Srivastava and L. Zamick, Minimal theory of isomerism- Q.Q and other interactions, Physica Scripta 96(6) (2021), 5307. https://doi.org/10.1088/1402-4896/abf306 
[10] M. Vygodsky, Mathematical Handbook, Moscow: Mir, 1978.

[11] L. Türker, A novel total $\pi$-electron energy formula for alternant hydrocarbons-angle of total $\pi$-electron energy, Match 30 (1994), 243-252.

[12] A. Graovac, I. Gutman and N. Trinajstic, Topological Approach to the Chemistry of Conjugated Molecules, Berlin: Springer-Verlag, 1977.

https://doi.org/10.1007/978-3-642-93069-0

[13] L. Türker, An approach to the construction of certain isospectral and subspectral graphs, Match 25 (1990), 195-215.

[14] I. Gutman and O.E. Polansky, Mathematical Concepts in Chemistry, Berlin: SpringerVerlag, 1986. https://doi.org/10.1007/978-3-642-70982-1 